\title{
Sistema de alerta para estudiantes con discapacidad visual en la UTM
}

\author{
Alert system for students with visual disabilities at the UTM
}

Sistema de alerta para estudiantes deficientes visuais en UTM

\author{
Marely del Rosario Cruz-Felipe ${ }^{*}$ \\ Jenmer Maricela Pinargote-Ortega ${ }^{1}$ \\ Gabriel Primitivo Demera-Ureta ${ }^{1}$ \\ Edisson Manuel Vera-Zambrano ${ }^{1}$ \\ Rosa Antonia Mosquera-Alcívar ${ }^{1}$
}

Recibido: agosto de 2017

Aceptado: noviembre de 2017

Para citar este artículo: Cruz-Felipe, M.R., Pinargote-Ortega, Demera-Ureta, G.P., Vera-Zambrano, E.M., Mosquera-Alcívar, R.A (2018). Sistema de alerta para estudiantes con discapacidad visual en la UTM. Revista Científica, 31(1), 85-95. Doi: https://doi.org/10.14483/23448350.12464

\section{Resumen}

El traslado de estudiantes con discapacidad visual en la Universidad Técnica de Manabí (UTM) ha reportado accidentes al pasar por algunas rampas y otros obstáculos, sobre todo en los días de lluvia. El presente artículo es parte de una investigación para la realización de un sistema de alerta para estudiantes con discapacidad visual. El objetivo de la implementación de este sistema es orientar a estudiantes con discapacidad visual sobre diferentes obstáculos que existen en su traslado por la universidad. Para llevar a cabo la implementación fueron analizados los sistemas de alertas y las tecnologías que se emplean actualmente como resultado de un profundo estudio del arte a nivel nacional e internacional; se describieron las herramientas y tecnologías utilizadas en la solución desarrollada (metodología, tecnologías para censar el desplazamiento de las personas, software, lenguajes de programación, etc.). Esto facilitó una implementación eficiente en corto tiempo del sistema propuesto mediante tecnología RFID (identificación por radio frecuencia). Lo anterior se refleja de manera positiva en la orientación para el traslado de 32 estudiantes con discapacidad visual por la universidad y, por consiguiente, mejora su calidad de vida.

Palabras clave: discapacidad visual, RFID, sistema de alerta.

\begin{abstract}
In the transfer of students with visual disabilities at the Technical University of Manabí (UTM) accidents have been reported when going through some ramps and other obstacles, especially on rainy days. This article is part of to an investigation into the realization of an alert system for students with visual disabilities. The objective of the implementation of this system is to guide students with visual disabilities on
\end{abstract}


different obstacles that exist in their transfer through the university. To carry out the implementation of this system, the alert systems and the technologies that are currently used as a result of a recording studio in the national and international scope were analyzed, the tools and technologies used in the developed solution are described. (Definition, technologies for the change of people, software, programming languages, etc.) that allowed an efficient implementation in a short time of the proposed system by means of RFID (Radio Frequency Identification) technology. The above is reflected in the positive orientation for the transfer of 32 students with visual disabilities through the university and by those who have contributed to improving their quality of life.

Keywords: visual disability, RFID, warning system.

\section{Resumo}

Na transferência de alunos com deficiência visual na Universidade Técnica de Manabí (UTM), foram notificados acidentes ao passar por algumas rampas e outros obstáculos, especialmente nos dias chuvosos. Este artigo é parte de a uma investigação para a realização de um sistema de alerta para estudantes com deficiência visual. O objetivo da implementação deste sistema é orientar estudantes com deficiências visuais em diferentes obstáculos que existem em sua transferência pela universidade. Para realizar a implementação deste sistema, foram analisados os sistemas de alerta e as tecnologias que atualmente são utilizadas como resultado de um estúdio de gravação no âmbito nacional e internacional, descrevendo as ferramentas e tecnologias utilizadas na solução desenvolvida. (Definição, tecnologias para mudança de pessoas, software, linguagens de programação, etc.) que permitiram uma implementação eficiente em um curto espaço de tempo do sistema proposto por meio da tecnologia RFID (Radio Frequency Identification). O acima se reflete na orientação positiva para a transferência de 32 alunos com deficiência visual através da universidade e por aqueles que contribuíram para melhorar sua qualidade de vida.

Palavras-chaves: deficiência visual, RFID, sistema de alerta.

\section{Introducción}

Los sistemas de alerta temprana (SAT) son un conjunto de dispositivos que determinan la posibilidad de una amenaza hacia una o más poblaciones. Esta anticipación se da mediante el monitoreo de una o varias señales físicas que intervienen en la amenaza. Los SAT resultan importantes en la prevención de pérdidas humanas y de bienes materiales de difícil recuperación (cultivos e infraestructura). Por medio de ellos se puede recolectar la información necesaria para el pronóstico de la ocurrencia y así facilitar la amortización de eventos futuros (Vargas, Tovar y Villanueva, 2016).

Estos SAT avisan con antelación un acontecimiento natural o humano que pueda causar un desastre con el objetivo de evitarlo. Desde la terminología de la gestión de riesgos, la dimensión del desastre está en la función de la fuerza del evento o en la prevención de accidentes y del nivel de vulnerabilidad de la población ante el mismo. El evento en sí no es necesariamente un desastre, algunos sistemas fueron diseñados y puestos en funcionamiento para avisar a la población de la proximidad de un evento y hacerlos mucho menos vulnerable de forma inmediata. Los SAT pueden tratar de anticipar los eventos naturales o de origen humano (generalmente conflictos violentos o prevenciones), que en interacción con la vulnerabilidad pueden desembocar en desastres.

La discapacidad visual consiste en la afectación o en la carencia de la visión, en mayor o menor grado. En sí misma no constituye una enfermedad, al contrario, es la consecuencia de un variado tipo de enfermedades. Al referirse a este tipo de discapacidad, Diez (2004) menciona que se trata de todas aquellas condiciones caracterizadas por una limitación total o parcial de la función visual que presenta un individuo.

Una persona con baja visión es aquella que presenta un rango menor a 20/400 o 0,05, considerando esta medición en el menor ojo y con la mejor corrección. En la ceguera total, por otro lado, su rango es menor a 20/200 o 0,1, con el mejor ojo 
y la mejor corrección. Es importante realizar esta diferenciación, pues la mayoría de personas consideradas ciegas presentan siempre un resto visual que le permite de una u otra forma desenvolverse y realizar actividades diarias (Suárez, 2011).

Dentro de la Universidad Técnica de Manabí (UTM) encontramos, en diferentes carreras, 36 estudiantes con discapacidad visual; dentro de las facultades con cifras más representativas de estos estudiantes está la Facultad de Ciencias Humanísticas y Sociales. En ella, estos estudiantes se deben desplazar por varios edificios como: una biblioteca que está orientada a sus necesidades, un edificio en el que se encuentran aulas y laboratorios y un bloque de edificios en el cual se encuentran algunos laboratorios, oficinas y teatro.

Para el traslado de un edificio a otro los estudiantes deben de acceder por varias escaleras y algunas rampas (las cuales han ocasionado lesiones a algunos de ellos, sobre todo en época de lluvia), además de los inconvenientes que representa no poderse orientar correctamente producto de su dificultad visual.

La universidad trabaja para que todos los estudiantes sean incluidos y puedan desarrollar todas sus actividades con el mismo éxito. Es por esto que se hace un estudio de las principales dificultades para el traslado de estudiantes con discapacidad visual en la Facultad de Ciencias Humanísticas y Sociales.

Ante el inconveniente de poder trasladarse sin dificultades de un edificio a otro, incluso orientarse correctamente (pues existen áreas en los edificios como las zonas de parqueo, los cuales pueden ser muy peligrosos para estos estudiantes), se hace necesario crear algún sistema que alerte a los estudiantes en sus desplazamientos y le sirva de ayuda incluso estando solos.

En el abanico de soluciones existentes para para ayudar a una persona a en su traslado se encuentran las que forman parte de la internet de las cosas, donde la conexión inalámbrica es la alternativa. Con base en la infraestructura existente, hay varias formas de conectar los objetos o cosascomo:
RFID, ZigBee, WPAN, WSN, DSL, UMTS, GPRS, WiFi, WiMax, LAN, WAN, 3G, etc. (Nitti, Atzori y Cvijikj, 2014). Partiendo del uso de tecnologías como las mencionadas, y otras como GPS, NFC, el empleo de la realidad aumentada y RFID, se han desarrollado una variedad de aplicaciones para la orientación de personas con discapacidad visual.

En la Universidad Tecnológica de Panamá se desarrolló una aplicación móvil denominada Teubica, la cual proporciona a las personas con discapacidad visual la posibilidad de conocer el lugar donde se encuentra al instalar una aplicación en el teléfono de otra persona al cual se envían algunas alertas de emergencia para ser ubicado en caso de que lo necesite. Este sistema emplea tecnología GPS (De Tristán et al., 2016).

Otros trabajos que involucran una tecnología incorporada para la movilidad de personas con discapacidad visual en los medios de transporte público son el OnTheBus y el App\&Town, los cuales funcionan en Barcelona y Alemania. Estos sistemas trabajan con tecnología GPS (Esteve, 2011; Once, 2012). También se ha desarrollado un sistema de posición y orientación móvil para personas ciegas en ambientes cerrados en la Universidad de Chile. Este sistema emplea tecnología WIFI (Sáenz, 2009).

El empleo de aplicaciones instaladas en teléfonos, como los mencionados anteriormente, requiere del uso de internet, lo cual representa una limitante. Sin embargo, la tecnología RFID brinda la posibilidad de no estar conectada a la red, además tiene una precisión mayor que las tecnologías GPS. Por tal motivo, en este trabajo se persigue como objetivo desarrollar un sistema de alerta a personas con discapacidad visual empleando tecnología RFID (identificación por radio frecuencia).

\section{Metodología}

Los métodos usados en esta investigación fueron cuatro y son descritos a continuación:

Inductivo-deductivo: a partir de la identificación de los problemas, el estudio y el análisis 
correspondiente del lugar a investigar, se obtuvo la información y con ello se procedió a las pruebas de los equipos informáticos para la futura implementación del sistema de alarma con tecnología RFID en la Facultad de Ciencias Humanísticas y Sociales de la UTM.

Analítico-sintético: este método facilitó la obtención de los elementos necesarios para realizar la implementación del sistema de alarma con tecnología RFID. Con este se logró identificar las necesidades del sistema y seleccionar una antena con tecnología RFID, etiquetas chip RFID y desarrollar el software (el cual, de acuerdo a la metodología, se organizó correctamente la implementación sin causar inconvenientes a los mismos).

Histórico-lógico: con este se hizo el estudio de los principales acontecimientos que han marcado la evolución de las tecnologías de internet de las cosas. Con ello se realizó el análisis correspondiente a las tecnologías asociadas como NFC, tecnologías de identificación, sistemas de localización geográfica y se determinó que la tecnología necesaria en este contexto es la RFID. Lo anterior permitió una mayor comprensión del marco teórico relacionado con los temas de estudio de la presente investigación científica como: el desarrollo de la tecnología RFID, los componentes, el alcance y la aplicación.

Modelación: en esta etapa el sistema fue modelado empleando las tecnologías que lo componen y se probó su funcionamiento como un todo; tales componentes y tecnologías son: lector RFID, antenas, etiquetas, se modela el comportamiento del middleware en la lectura de las etiquetas, la actualización de la base de datos, el módulo de gestión de los usuarios y el comportamiento de la aplicación para el móvil en el que se alerta a través de un mensaje de voz a los usuarios.

\section{Métodos empíricos}

Las herramientas metodológicas de carácter empírico que fueron usadas en la investigación son:
- Entrevistas a estudiantes con discapacidad visual de la UTM, los cuales han aportado elementos significativos a la investigación. Los resultados han permitido detectar a partir de la realidad cuáles son las necesidades de estos estudiantes y las características de los mismos, los tipos de discapacidad visual que tienen, las posibilidades de empleo de un teléfono móvil, las principales dificultades que afrontan en el traslado. Esta última información sirvió para seleccionar los obstáculos más significativos y tenerlos en cuenta en la ubicación de las antenas, de modo tal que el sistema funciones de la forma más óptima posible.

- Análisis de documentos y bibliografías referentes al tema con los cuales se extrajo el estado actual de las tecnologías a emplear como los sistemas RFID y la definición del uso de las mismas.

\section{Resultados}

Los resultados del sistema de alerta a estudiantes con discapacidad se centran en incorporar un conjunto de tecnologías y herramientas elaboradas para abarcar todas las necesidades para la movilidad de este tipo de estudiantes. Para esto, se describen a continuación las tecnologías y herramientas y luego se muestra el sistema de alerta haciendo hincapié en lo que aporta cada una de las herramientas desarrolladas.

\section{Selección de tecnologías, lenguajes de program- ación y herramientas}

Metodología de desarrollo Extreme Programming (XP)

A partir del análisis de las diferentes metodologías de desarrollo existentes, se seleccionó la XP ya que es una metodología ágil que permitió planificar en corto tiempo el desarrollo del sistema de alerta con un diseño simple, en el que se trabajó en equipo y en iteraciones con sus respectivas 
pruebas. Misma que permitirá a futuro hacer otros incrementos al sistema.

Lenguaje de programación

Java. Además se hizo uso de herramientas de Eclipse Mars, JavaJDK1.7, Genymotion, PHP, TOMCAT, Netbeans y JDK 1.7

\section{Android Studio}

Se empleó para el desarrollo de la aplicación móvil.

\section{Servidor XAMPP}

Administrador del servidor Apache.

Sistema de gestión de bases de datos (MySQL)

Con el editor de base datos SQL Manager 2007 for MySQL se creó una base de datos llamada "rfid_ database", compuesta por cinco tablas, en la que cada una cumple la función de registro de datos.

\section{Web Service}

Permitió mantener una comunicación entre todas las aplicaciones creadas en distintas plataformas de programación como php, java, android y mysql; además, con ayuda de este sistema y también de la programación, se pudo realizar el cambio del mensaje de texto a mensaje de voz.

\section{Selección de la tecnología de identificación para en el sistema de alarmas}

Se realizó el análisis de las diferentes tecnologías de identificación. En ellas se parte del empleo de algún dispositivo móvil que la persona con discapacidad pudiera portar, para esto se analiza el empleo de la tecnología NFC (Near Field Communication, o Comunicación de Campo Cercano en español). La comunicación que se establece entre los dos dispositivos debe realizarse desde una distancia reducida, ya que el alcance de la tecnología es limitado a un máximo de $20 \mathrm{~cm}$ (NFC Forum, 2017). Usualmente, NFC trabaja con una frecuencia de $13.56 \mathrm{MHz}$ y una taza de transferencia de 424Kb/seg (Langer, Saminger y Grunberger, 2009; Acosta, Lozano y Rico, 2015). Lo cual no es factible en esta solución por la necesidad de acercarse a los sensores y la poca posibilidad que tendría una persona con discapacidad visual para emplearla por la distancia a la cual.

Otra de las posibles soluciones es la de utilizar un sistema GPS, pero este tiene como inconveniente el empleo de un sistema cartográfico (el cual es muy costoso) y la poca precisión si se trata de distancias de pocos metros.

En seguida, se analiza la tecnología RFID, la cual es ampliamente empleada para censar objetos (desde inanimados hasta personas). A través de ella se detecta la etiqueta mediante un lector y se identifica esta. Esta tecnología permite la lectura de la etiqueta a una distancia del orden de los metros.

Por último, se analizó dentro de la tecnología RFID los lectores y antenas que soportan un rango de $3 \mathrm{~m}$ de distancia; los mismos trabajan en el rango de frecuencias UHF. Se seleccionaron e instalaron los siguientes equipos que cumplen con estas características: FX7400-2 Port Fixed RFID Reader GEN2 POE, AN480 RFID Antenna High PERF LH CIRC POL NO, cable 240- LMR-240TNC/N, por cada antena RFID se utilizó 25 mts de cable y etiquetas CHIP RFID. Lo que permitió realizar la demostración del sistema de alarma para personas no videntes con la utilización de la tecnología RFID.

\section{Sistema de alerta}

De acuerdo a la investigación realizada, se obtuvo un sistema que consta decuatro herramientas: una para la gestión de usuarios, Ilamada Aplicación RFID, una aplicación móvil, un middleware y una base de datos.

La aplicación RFID es una herramienta desarrollada para: 
- Gestionar el personal. En esta aplicación es posible introducir los datos personales de los usuarios (en este caso, estudiantes con discapacidad visual), además del número de teléfono, que será empleado para vincular la herramienta RFID con una que se instala en el teléfono cuyo número se registra en esta aplicación.

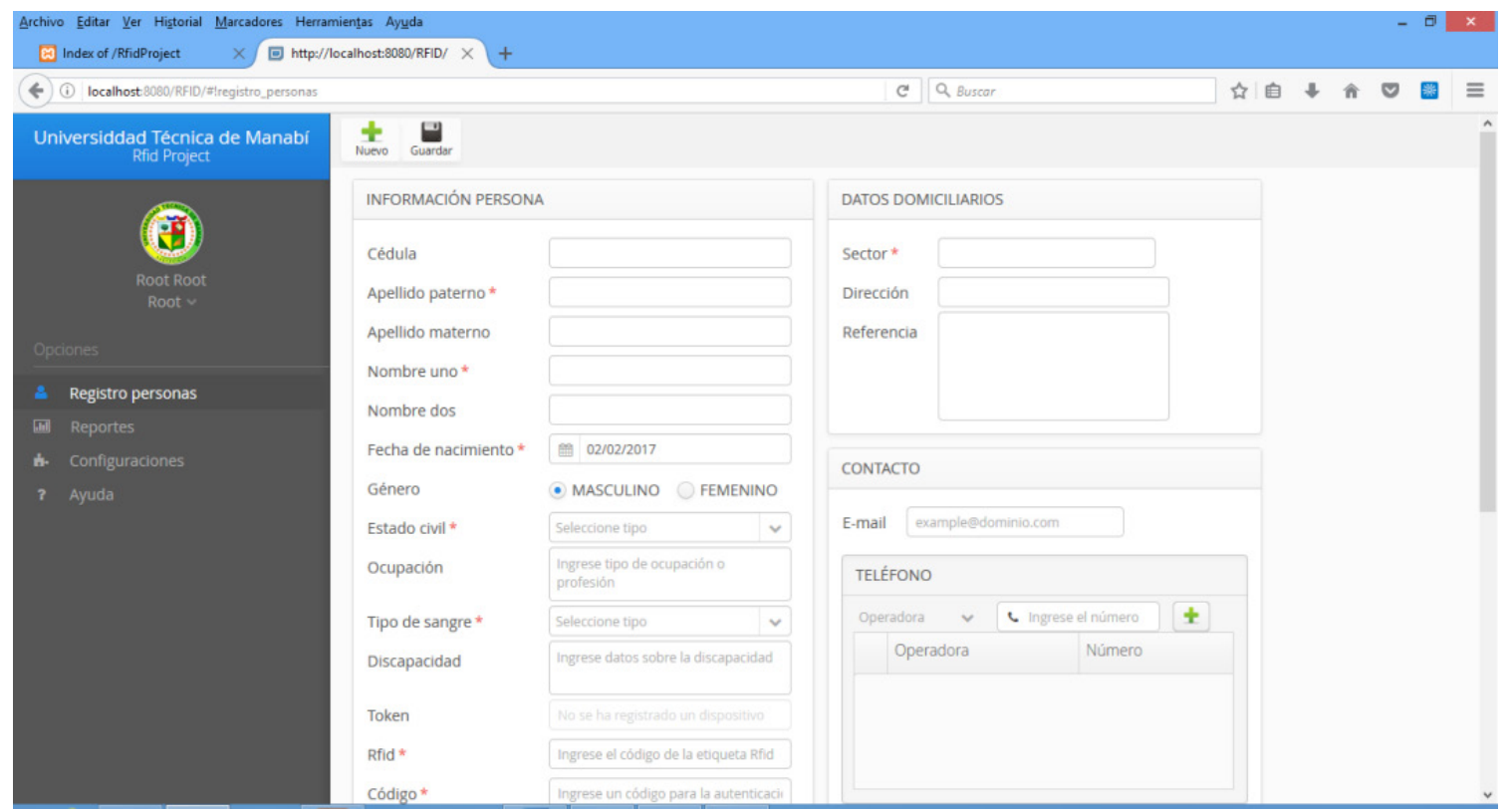

Figura 1. Gestionar personal.

Fuente: elaboración propia.

- La realización de reportes.

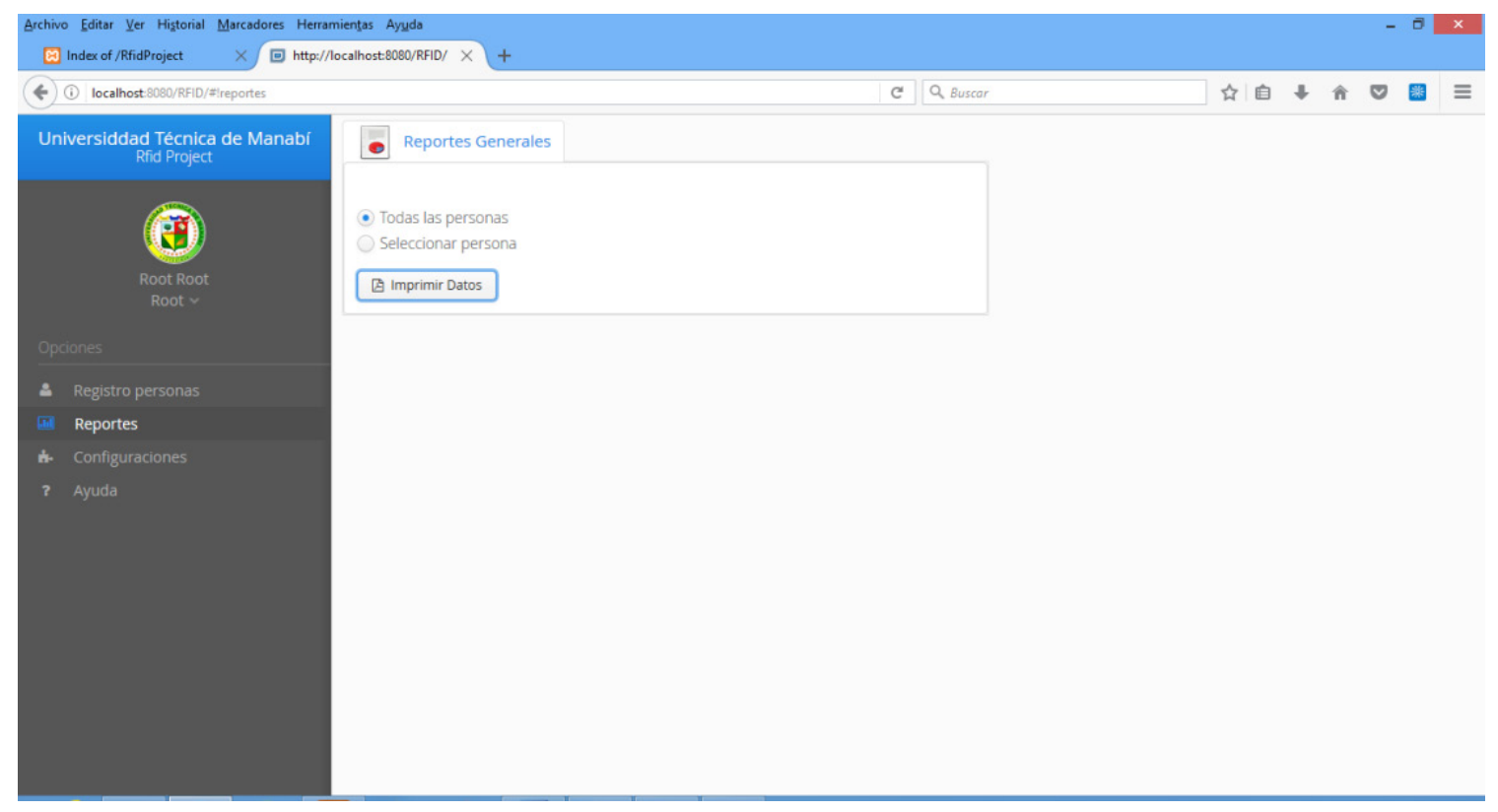

Figura 2. Opción Reportes.

Fuente: elaboración propia. 
- La selección de la ubicación de cada usuario.

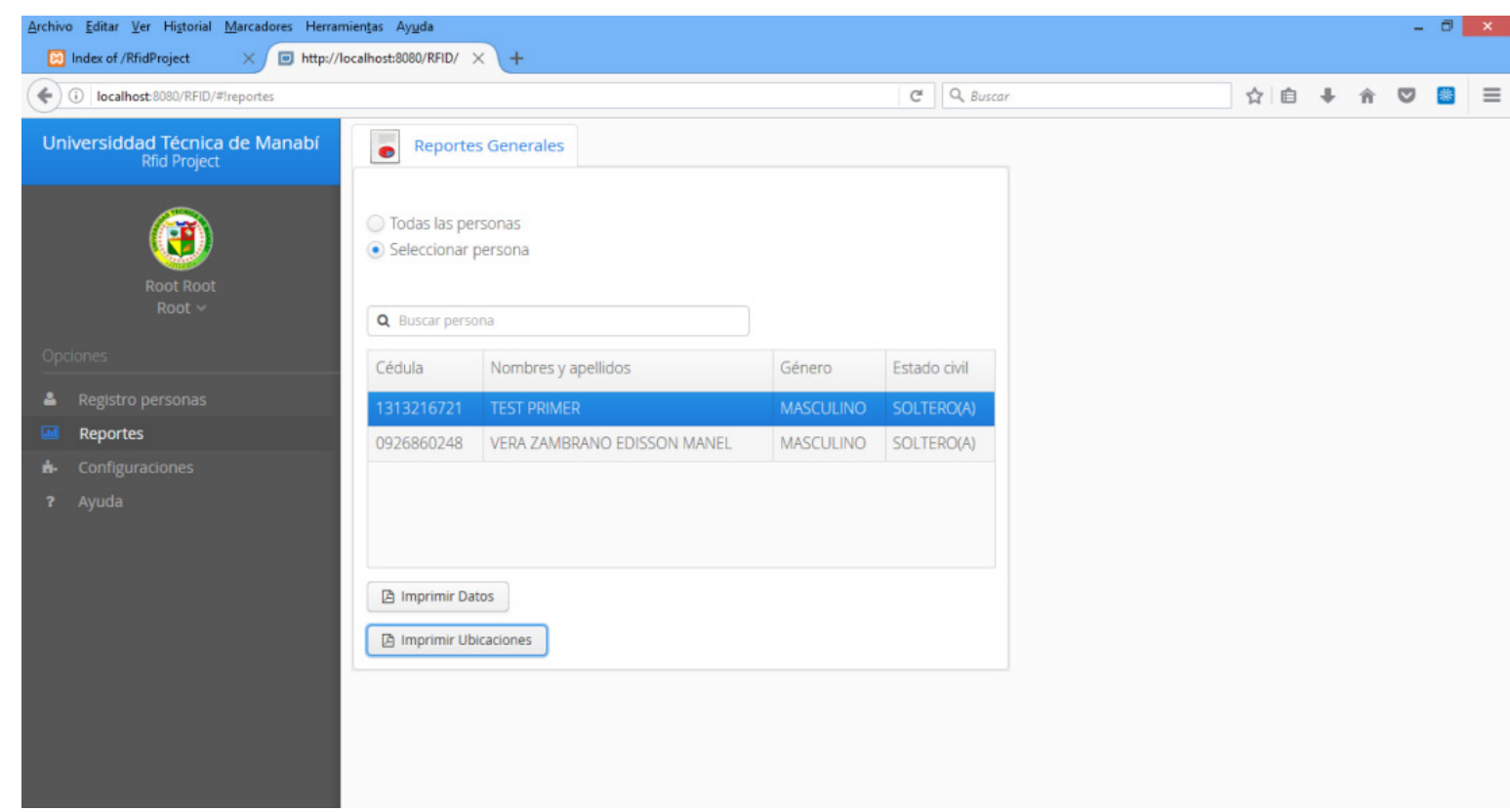

Figura 3. Gestión de usuario.

Fuente: elaboración propia.

- Configuración de antena a teléfono celular. En esta sección se configura la antena de acuerdo a la ubicación que tenga al editar un texto con los datos de los obstáculos que se encuentran cerca de la misma, el cual será enviado en forma de texto a la aplicación del teléfono.

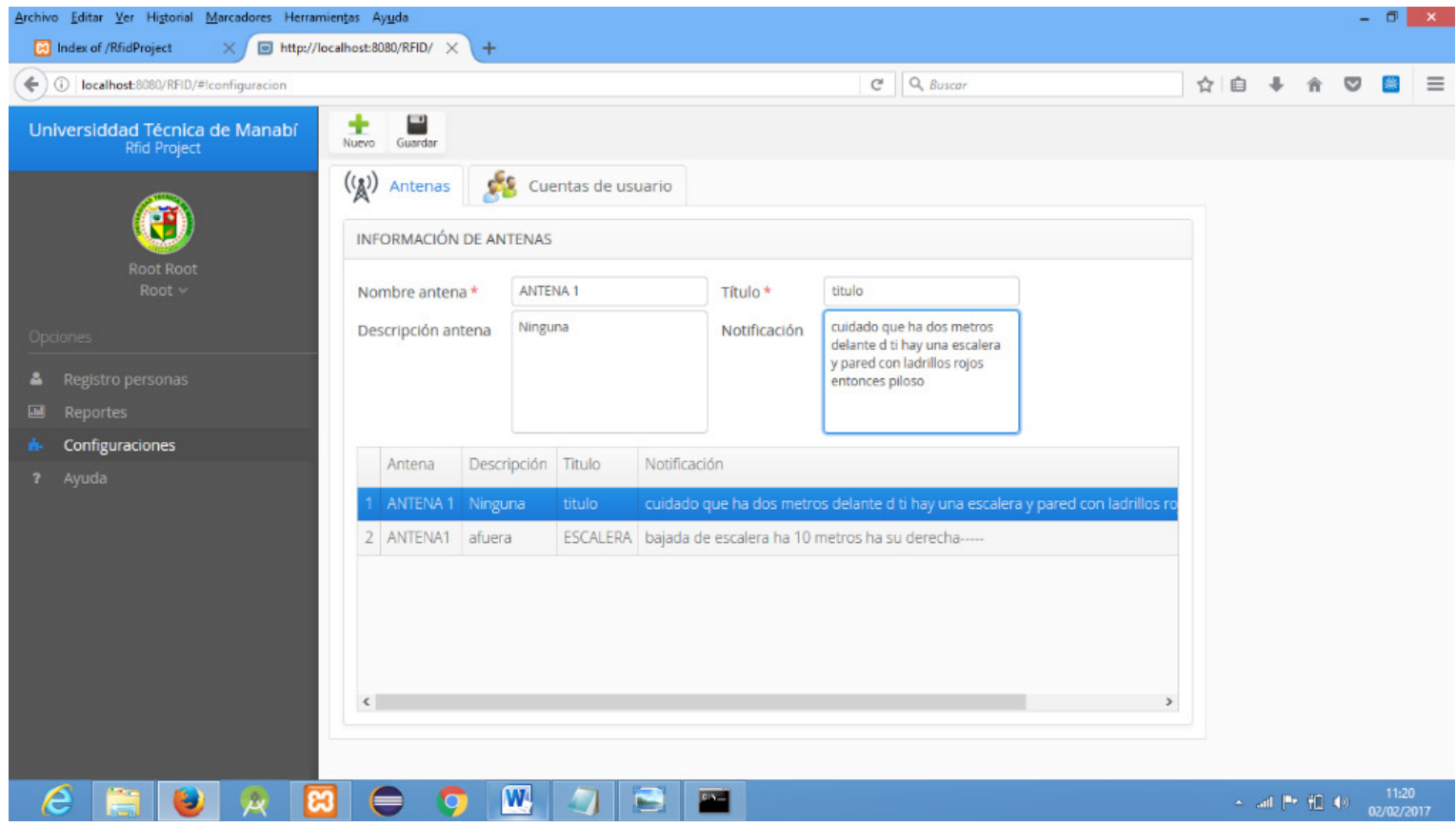

Figura 4. Configuración de antena a teléfono celular.

Fuente: elaboración propia. 
- La aplicación Middleware permite el monitoreo en tiempo real de las personas mediante la lectura de las etiquetas RFID. Esta aplicación además permite filtrar el tráfico y enviar a la aplicación RFID los datos de las etiquetas que han sido leídas a través de la antena y el lector.

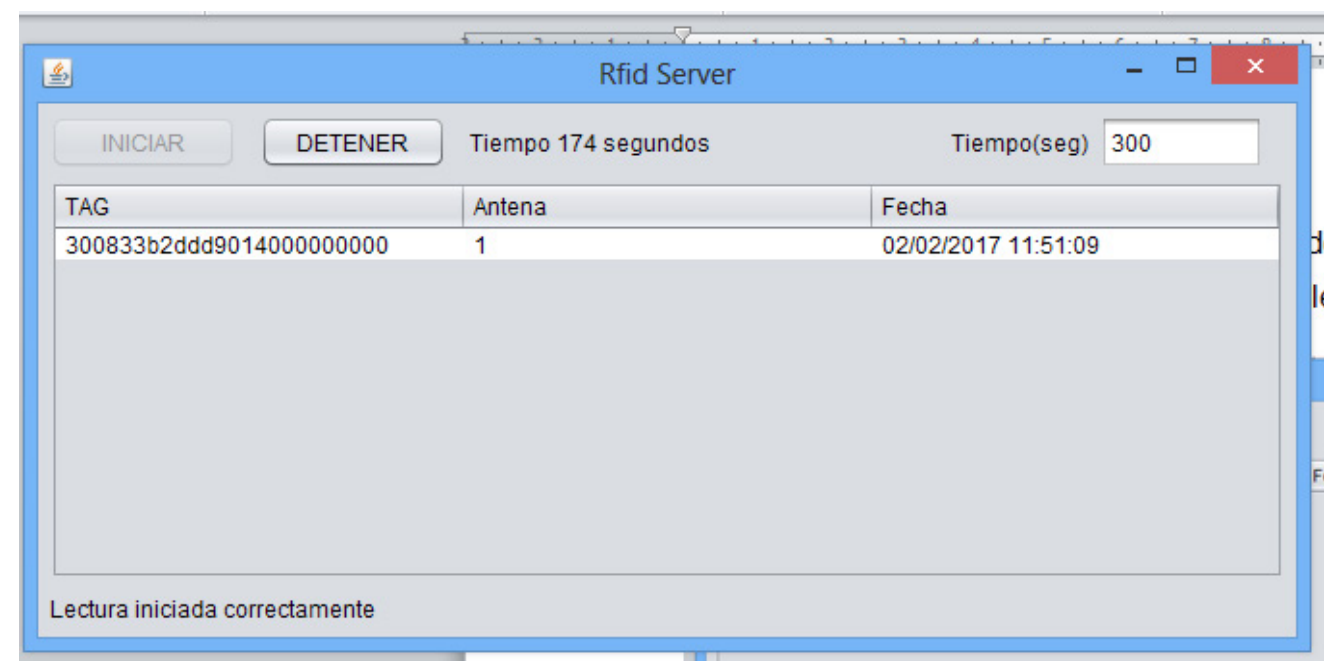

Figura 5. Prueba de lectura de las etiquetas.

Fuente: elaboración propia.

- La aplicación móvil es instalada en el teléfono, en esta se inicia sesión y se recibe el audio. Esta aplicación brinda la posibilidad a la persona no vidente de escuchar un audio que le alerta del obstáculo que se encuentre cercano a su posición.

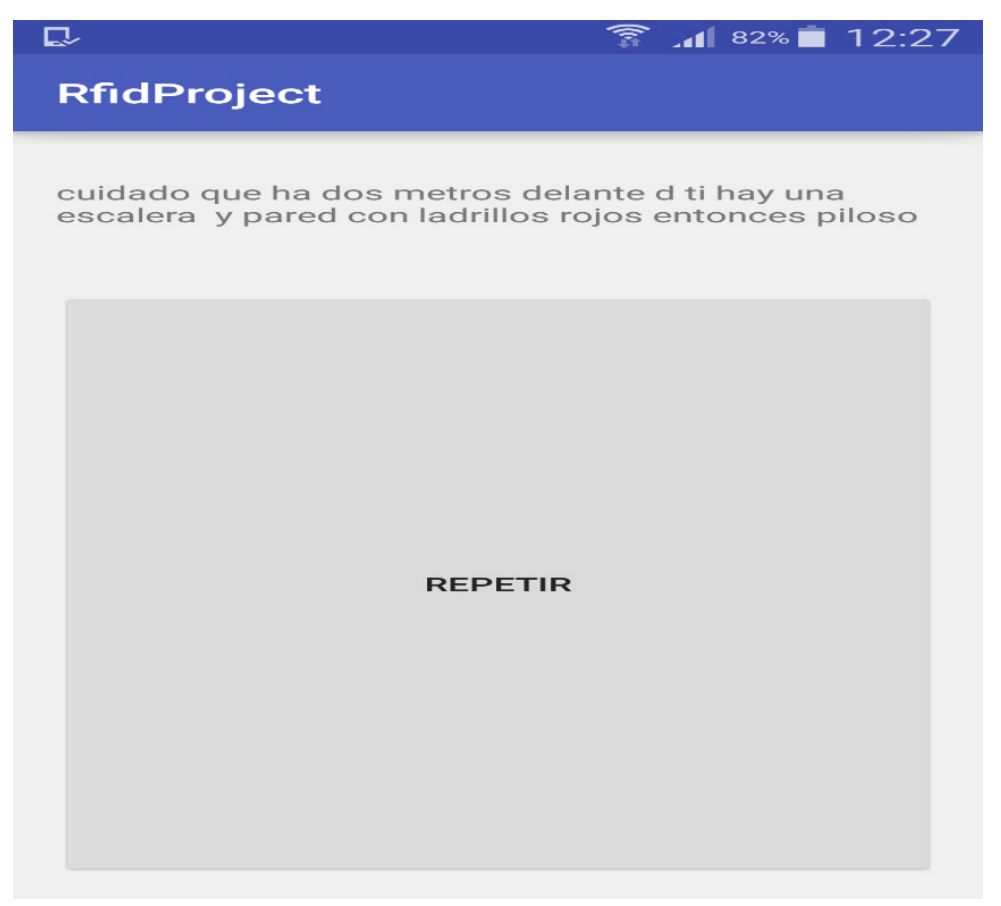

Figura 6. Aplicación instalada en el teléfono móvil.

Fuente: elaboración propia. 


\section{Modelo de base de datos}

En la base de datos creada en MYSQL se almacenan los mensajes de voz, los datos de los usuarios que tienen el acceso al sistema, datos de las antenas, los código de cada etiqueta y lo más importante: los datos de las personas con discapacidad visual. En esta base de datos se lleva un registro de la hora, fecha y lugar por el que pasó cada persona con discapacidad visual que posee una etiqueta ya registrada.

\section{Arquitectura del sistema}

El despliegue del sistema está compuesto por un servidor, un teléfono móvil, un lector y la antena, los cuales deberán estar conectados a una misma red. Puesto que cada antena y lector tienen una dirección IP dada por el fabricante que es estática.

Al momento de conectarnos a la red de la universidad, por defecto esa red va a generar una dirección IP privada y esto causaría dificultad; otra conflicto que se tendría en una red de la universidad es el número del puerto por el cual se va a conectar con los equipos, que estaría posibilitando trabajar en diferentes números de puerto y en este sistema es necesario trabajar con el puerto "8080". Por lo que la solución a esto es trabajar en una red definida solo para este sistema lo que permitiría definir un nuevo puerto.

Para este punto se implementó un router de 5 puertos con conexión LAN y distribución de internet inalámbrico, las conexiones por medio del cable de red son para conectar el lector y el servidor, y la conexión inalámbrica sirve para conectar el teléfono, y para la conexión por medio de internet al web service, lo que permite mantener una comunicación de todos los equipos en una sola red y funcionando al $100 \%$.

Una arquitectura completa del sistema se muestra en la figura 8.

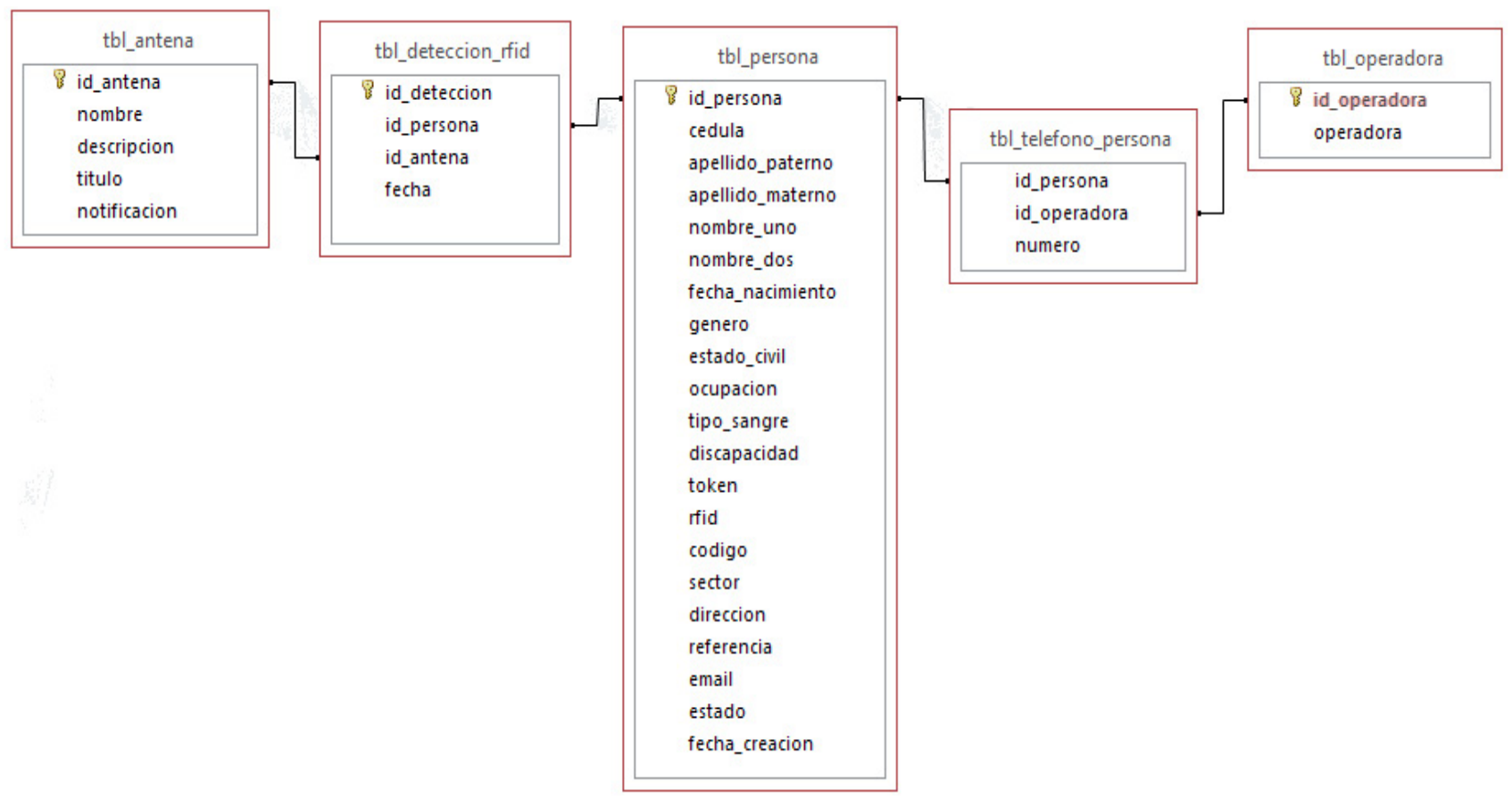

Figura 7. Modelo de base de datos.

Fuente: elaboración propia. 


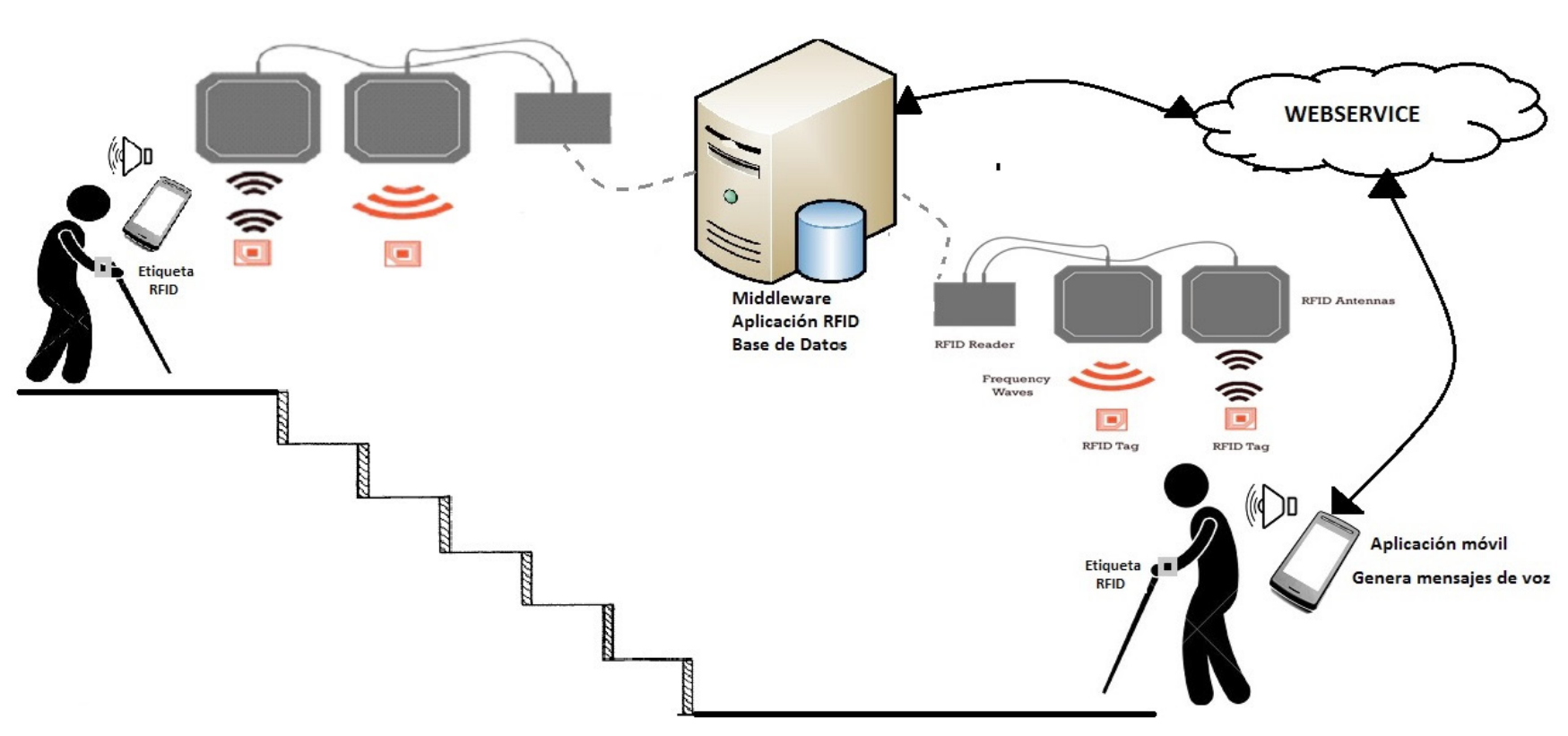

Figura 8. Arquitectura del sistema.

Fuente: elaboración propia.

Desde la descripción de la arquitectura del sistema se demuestra cómo se integran diferentes tecnologías y se crean tres aplicaciones que permiten darle solución al problema planteado. A partir de las pruebas de usuarios y configuración del sistema, se demostró el correcto funcionamiento del sistema obtenido.

\section{Conclusiones}

Se obtiene un sistema de alarma en el cual se elabora e integran una aplicación web, un middleware, una base de datos y una aplicación móvil. Estas herramientas han sido desarrolladas con código abierto, lo que permitirá realizar futuras ampliaciones del sistema. En el sistema propuesto, la aplicación web permite la gestión de usuarios del sistema, almacenando todos los datos de las personas con discapacidad, la realización de reportes, la ubicación de las personas no videntes, la configuración de la antena RFID al teléfono celular, la lectura de las etiquetas y la inicialización del sistema y recepción de una señal de audio. El empleo del sistema de alarma obtenido permite la orientación de las personas no videntes de la Facultad de Ciencias Humanísticas y Sociales de la UTM, lo cual reduce la posibilidad de accidentes por obstáculos como escaleras, rampas y parqueaderos que se encuentran en el área a trasladarse dentro de la universidad. Todo esto mejora la calidad de vida de un grupo considerable de estudiantes que presentan discapacidad visual en la UTM.

\section{Referencias}

Acosta López, A., Lozano, H.D. y Rico, C. (2015). Diseño e implementación de un prototipo de sistema de identificación por radiofrecuencia para la verificación de información de vehículos. Redes de Ingeniería, 6(2), 33-43. https://doi. org/10.14483/udistrital.jour.redes.2015.2.a04

De Tristán, G., Arcia, A., Pérez, R. y Montes, H., (2016). Aplicación móvil para el monitoreo de personas con discapacidad visual. Conferencia presentada en 7 th International Congress on Application of Advanced Information and Communications Technologies (Atica), Cuenca, Ecuador, vol. 1. 
Diez, A.C. (2004). Las necesidades educativas especiales: políticas educativas en torno a la alteridad. Cuadernos de antropología social, 19, 157-171.

Esteve, M. (2011). OnTheBus (proyecto de fin de carrera). Universidad Autónoma de Barcelona, Barcelona.

Langer J., Saminger C. y Grunberger S. (2009). A comprehensive concept and system for measurement and testing Near Field Communication devices. IEEE Regions \& Conference EUROCON, 2052-2057. https://doi.org/10.1109/ EURCON.2009.5167930

NFC Forum. (2017). About the Technology. NFC and Contactless Technologies. Recuperado de: http://nfc-forum.org/what-is-nfc/ about-the-technology/.

Nitti M., Atzori L. y Cvijikj I.P. (2014). Network Navigability in the Social Internet of Things.
IEEE World Forum on Internet of Things (WF-loT), 405-410. https://doi.org/10.1109/ WF-IoT.2014.6803200

Once. (2012). Aplicación Once-Cidat Metro para IPhone. Centro de Investigación, Desarrollo y Aplicación Tiflotécnica de la Once (Cidat).

Sáenz, M.A. (2009). Sistema de posición y orientación móvil para personas ciegas en ambientes cerrados (tesis de maestría). Facultad de Ciencias Físicas y Matemáticas, Departamento de Ciencias de la Computación, Universidad de Chile, Santiago.

Suárez, J.C. (2011). Discapacidad visual y ceguera en el adulto: revisión de tema. Medicina U.P.B., 30(2), 170-180.

Vargas, H.F., Tovar, M.F. y Villanueva J.C. (2016). Los SAT (sistemas de alertas tempranas). Revista Científica, 26, 21-28. https://doi. org/10.14483/23448350.11087 